\title{
Microstructure sensitive design of an orthotropic plate subjected to tensile load
}

\author{
Brent L. Adams \\ b_I_adams@byu.edu \\ Mark Lyons \\ Joshua R. Houskamp \\ Surya R. Kalidindi
}

Follow this and additional works at: https://scholarsarchive.byu.edu/facpub

Part of the Mechanical Engineering Commons

Original Publication Citation

International Journal of Plasticity 2 (24) 1561-1575

\section{BYU ScholarsArchive Citation}

Adams, Brent L.; Lyons, Mark; Houskamp, Joshua R.; and Kalidindi, Surya R., "Microstructure sensitive design of an orthotropic plate subjected to tensile load" (2003). Faculty Publications. 494.

https://scholarsarchive.byu.edu/facpub/494

This Peer-Reviewed Article is brought to you for free and open access by BYU ScholarsArchive. It has been accepted for inclusion in Faculty Publications by an authorized administrator of BYU ScholarsArchive. For more information, please contact ellen_amatangelo@byu.edu. 


\title{
Microstructure sensitive design of an orthotropic plate subjected to tensile load
}

\author{
Surya R. Kalidindi a,*, Joshua R. Houskamp a, \\ Mark Lyons ${ }^{b}$, Brent L. Adams ${ }^{b}$ \\ a Department of Materials Science and Engineering, Drexel University, Philadelphia, PA, USA \\ ${ }^{\mathrm{b}}$ Department of Mechanical Engineering, Brigham Young University, Provo, UT, USA
}

\begin{abstract}
Microstructure Sensitive Design (MSD) is a newly developed mathematical framework that facilitates rigorous solutions to inverse problems in microstructure design of materials. In this paper, this methodology is applied to an orthotropic thin plate containing a circular hole subjected to an in-plane uniaxial tensile load. The primary design objective is to maximize the load carrying capacity of the plate while avoiding plastic deformation in the plate. Making use of the inherent anisotropy of fcc polycrystals arising from distribution of lattice orientations (also referred to as crystallographic texture), microstructures have been identified in copper that are predicted to yield the best and worst possible performance, respectively. The microstructure with the best load carrying capacity was found to show an increase of about $59 \%$ compared to the microstructure with the worst load carrying capacity. The solutions from the MSD methodology were validated by direct comparisons from finite element simulations that employed a Taylor-type polycrystal constitutive model at each integration point. A reasonable agreement was obtained between MSD predictions and finite element simulations.
\end{abstract}

\section{Introduction}

In recent years, the discipline of materials science has witnessed the emergence of a grand challenge that had always implicitly defined this multidisciplinary field, namely, the ability to identify and process specific materials and microstructures that

\footnotetext{
${ }^{*}$ Corresponding author. Tel.: +1-215-895-1311; fax: +1-215-895-6760

E-mail address: skalidin@cbis.ece.drexel.edu (S.R. Kalidindi).
} 
are predicted to meet or exceed multiple properties/performance criterion stipulated by the designer. Olsen $(1997,2000)$ labeled this new paradigm as the "goals-means" approach. This contrasts sharply with the traditional "cause and effect" approach that is driven mainly by innovations in processing, which typically focuses only a limited number of readily manufacturable microstructures. This inverted paradigm of materials design is especially critical for highly constrained design (HCD) applications, where the designer faces increasingly complex requirements with multiple property objectives/constraints and material anisotropy affecting system performance. It is in this context that the authors and their collaborators have recently proposed a new microstructure design framework called Microstructure Sensitive Design (MSD) (Adams et al., 2001).

MSD comprises a novel methodology to identify the best of all possible microstructures within a given mechanical and physical framework that meet the stipulated design performance requirements. Thus, information flow is in the 'inductive' direction: design objectives $\rightarrow$ properties $\rightarrow$ microstructure $\rightarrow$ processing. A dominant characteristic of MSD is its use of spectral representations in all aspects of the problem. These representations of microstructure can be used with established homogenization theory to estimate the effective properties of the material, and to frame the design requirements. The tensorial nature of spectral representations of microstructure and microstructure/properties relations insures that an optimally compact and complete representation of microstructure is used-no more and no less than what is precisely needed for the representation of the problem. The reader is referred to an earlier paper (Adams et al., 2001) for further details of the technique, where the example of the optimal design of material microstructure for a compliant beam component was worked out in detail. In the present paper, a new case study is presented that centers on the design of the optimal microstructure for a thin orthotropic plate with a small circular hole subjected to an in-plane tensile load. This case study expands the scope of MSD over the previous case study (Adams et al., 2001) in at least two ways: (i) this case study involves a multi-axial stress state whereas our previous case study involved only a uniaxial stress state imposed at the macroscale, and (ii) we present verification of the MSD predictions for the best possible performance by direct comparisons with corresponding finite element simulations.

\section{The design problem-plate with a circular hole}

Holes and notches occur frequently in engineering design. Special attention is paid in designing components containing notches or holes, since it is well established that they have a major effect on design life of the components, especially in fatigue loading. Mechanical design handbooks such as: CRC Materials Science and Engineering Handook, (Alexander, 2001), Handbook of Materials for Product Design (Harper, 2001), and SAE Fatigue Design Handbook (SoAEFDaE, 1997) depict a number of charts providing estimates of stress concentration factors that depend on the geometry of the notch. However, in virtually all of the design handbooks, the 


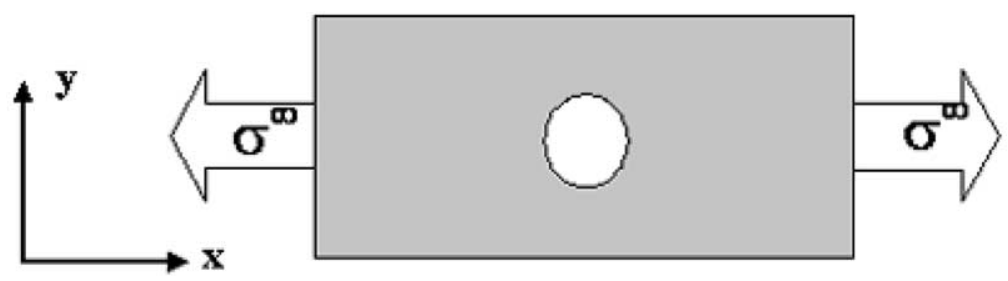

Fig. 1. Schematic of a plate with a circular hole loaded in tension.

material is assumed to possess isotropic mechanical properties. This practice, however, is in direct conflict with the trends in new materials development, where one of the major goals has been to enhance properties of the material in certain directions while sacrificing properties in other directions where they are not as needed (e.g. development of laminated composite systems). Anisotropy of the material is bound to play an extremely important role in highly constrained design (HCD) applications. In design of components with notches and holes, a relatively small reduction of the stress concentration factor can lead to a significant increase in the design life of the component (e.g. a $20 \%$ reduction in stress concentration factor can produce a $900 \%$ increase in the high-cycle fatigue life of a component made from SAE 4340 steel). In this preliminary case study, we explore the design of a thin orthotropic plate with a circular hole subjected to in-plane tensile load.

The primary objective in this design (see Fig. 1) is the maximization of the load carrying capacity, $\sigma^{\infty}$. The plate must provide this load carrying capacity without plastic yielding (primary constraint). Thus, both elastic and yielding properties are germane to the problem.

In this case study, we restrict our attention to only the orientational variables of the microstructure and their volume fractions (i.e. crystallographic texture). Note that the MSD framework, in principle, is capable of including other microstructural state variables (e.g. compositions) and higher-order spatial correlations [e.g. twopoint correlations of orientations and compositions (Adams et al., 2001)]. Introduction of these other features does increase significantly the computational load, and will be explored in future studies.

\section{Mechanical and physical framework of the design problem}

The stress distribution around a circular hole in a thin orthotropic plate, was derived by Lekhnitskii (1968) to be circumferential in nature, and given by

$$
\begin{aligned}
& \sigma_{\theta \theta}=\sigma^{\infty} \frac{E_{\theta}}{E_{x}}\left[-\sqrt{\frac{E_{x}}{E_{y}}} \cos ^{2} \theta+(1+n) \sin ^{2} \theta\right] \\
& n=\sqrt{\frac{E_{x}}{G_{x y}}-2 v_{x y}+2 \sqrt{\frac{E_{x}}{E_{y}}}}
\end{aligned}
$$




$$
\frac{1}{E_{\theta}}=\frac{\sin ^{4} \theta}{E_{x}}+\left(\frac{1}{G_{x y}}-\frac{2 v_{x y}}{E_{x}}\right) \sin ^{2} \theta \cos ^{2} \theta+\frac{\cos ^{4} \theta}{E_{y}}
$$

where $\theta$ is the angle measured from the $x$-axis, $\sigma^{\infty}$ is the stress applied at the edge of the plate (in the $x$-direction), $G_{x y}$ and $v_{x y}$ are shear modulus and Poisson's ratio in the $x$ - $y$ plane, and $E_{x}$ and $E_{y}$ are Young's moduli in the $x$ - and $y$-directions, respectively. Note that only four elastic constants $\left(E_{x}, E_{y}, G_{x y}\right.$, and $\left.v_{x y}\right)$ appear in this plane-stress design problem, and that their values are dictated by the details of the material microstructure.

Next, the orthotropic Hill yield description (Jones, 1999) is used to assess the initiation of plastic deformation in the plate. For plane stress conditions, it reduces to

$$
(G+H) \sigma_{x x}^{2}+(F+H) \sigma_{y y}^{2}-2 H \sigma_{x x} \sigma_{y y}+2 N \sigma_{x y}^{2}=1
$$

where $G, H, F$, and $N$ are orthotropic yield strength parameters that depend on the details of the material microstructure. Substituting the solution for the stress field around the hole [Eqs. (1-3)] into Eq. (4) yields

$$
\sigma_{\theta \theta}^{2}\left\{(G+H) \cos ^{4} \theta+(F+H) \sin ^{4} \theta+2(N-H) \sin ^{2} \theta \cos ^{2} \theta\right\}=1
$$

Note that, based on Eq. (4), the terms $(G+H)$ and $(F+H)$ can be related to uniaxial yield strengths of the material in $x$ - and $y$-directions, respectively, and $N$ can be related to the shear yield strength of the material in the $x-y$ plane. Note also that the equi-biaxial strength of the plate loaded in $x$ - and $y$-directions is related to $(G+F)$ and therefore one can derive an expression for $H$ in terms of the uniaxial yield strengths in $x$-and $y$-directions and the biaxial yield strength.

In summary, the engineering performance of the plate in this design is controlled by four elastic parameters $\left(E_{x}, E_{y}, G_{x y}, v_{x y}\right)$ and three plastic yield parameters $(G+H, F+H, N-H)$. For given values of these seven parameters, Eqs. (1)-(5) provide an expression for $\sigma^{\infty}$ as a function of $\theta$. The load carrying capacity is simply determined by the minimum value of $\sigma^{\infty}$ that satisfies Eq. (5) anywhere in the range $\theta \in[0,90]$. The seven parameters listed above control the performance of the plate, and are in turn strongly dependent on the details of the microstructure.

The microstructural design variable selected here is the one-point statistics (i.e. volume fractions) of the distribution of crystal lattice orientations (i.e. crystallographic texture). Therefore it is necessary to develop relationships between the macroscale elastic and plastic properties listed above and crystal orientations and their (statistical) distributions.

The fourth-rank elastic stiffness of a cubic crystal in its own reference frame (aligned with the [100] directions) can be expressed as

$$
C_{i j k l}^{\mathrm{c}}=\mathrm{C}_{12} \delta_{i j} \delta_{k l}+C_{44}\left(\delta_{i k} \delta_{j l}+\delta_{i l} \delta_{j k}\right)+\left(C_{11}-C_{12}-2 C_{44}\right) \sum_{r=1}^{3} \delta_{i r} \delta_{j r} \delta_{k r} \delta_{l r}
$$


where $C_{11}, C_{12}$, and $C_{44}$ are material constants representing the independent elastic constants in the local frame for cubic symmetry, and $\delta_{i j}$ represents the Kronecker delta function. The same elastic stiffness can be expressed in the fixed global reference frame (attached to the sample) as ${ }^{1}$

$$
C_{i j k l}=g_{i p} g_{j q} g_{k r} g_{l s} C_{p q r s}^{\mathrm{c}}
$$

where $g_{i j}$ represents the rotation matrix that transforms the crystal reference frame to the global reference frame. This transformation can be accomplished as a sequence of three rotations, and the set of the three rotation angles associated with these rotations can be represented conveniently as a set of Euler angles (Bunge, 1993). In other words, the lattice orientation of a crystal, $g$, can be represented equivalently either by the set of Euler angles $\left(g=\left[\phi_{1}, \Phi, \phi_{2}\right]\right)$ or by the rotation matrix $g_{i j}$, and their interrelationships are well established in literature (Bunge, 1993). The upper bound theory (Adams et al., 2001) is employed to obtain the elastic stiffness of the polycrystal representing the material microstructure.

$$
\bar{C}_{i j k l}=\oiint_{M_{\mathrm{c}}} f(g) C_{i j k l}(g) \mathrm{d} g
$$

In Eq. (8), $f(g)$ represents the orientation distribution function (ODF), and represents the normalized probability density of finding a crystal of orientation $g$ (Bunge, 1993). The integral in Eq. (8) is performed over the set, $M_{\mathrm{c}}$, comprising all possible single crystal orientations (taking into account cubic symmetry of the crystals); this set is called the material set.

Note that for a given microstructure, the elastic constants needed for the design of the orthotropic thin plate (namely $E_{x}, E_{y}, G_{x y}$, and $v_{x y}$ ) can be obtained from $\bar{C}_{i j k l}$, using established relations in literature (Jones, 1999).

For plastic yield properties, the overall approach taken here is to use a Taylortype crystal plasticity model and extract the relevant properties for the design $(G, F$, $H$, and $N$ ) from the predictions of the crystal plasticity model for a given microstructure. The details of the crystal plasticity model (Kalidindi et al., 1992) used in this study are briefly reviewed next, using a notation which is now standard in modern continuum mechanics. In particular, the deformation gradient is denoted by $\mathbf{F}$, the velocity gradient by $\mathbf{L}$, and the symmetric Cauchy stress by $\mathbf{T}$.

Assuming small elastic stretches but large plastic deformations, the equation for stress at a material point in a single crystal is expressed as

$$
\mathbf{T}^{*}=\mathbf{C}\left[\mathbf{E}^{*}\right], \quad \mathbf{E}^{*}=1 / 2\left\{\mathbf{F}^{* \mathrm{~T}} \mathbf{F}^{*}-1\right\}, \quad \mathbf{T}^{*}=\mathbf{F}^{*-1}\left\{\left(\operatorname{det} \mathbf{F}^{*}\right) \mathbf{T}\right\} \mathbf{F}^{*-\mathrm{T}}
$$

where $\mathbf{C}$ is the fourth order elasticity tensor described earlier [Eq. (7)], and $\mathbf{F}^{*}$ is an elastic deformation gradient defined in terms of the deformation gradient $F$ and a plastic deformation gradient $\mathbf{F}^{\mathrm{p}}$ with det $\mathbf{F}^{\mathrm{p}}=1.0$ (plastic incompressibility), by

$$
\mathbf{F}^{*}=\mathbf{F F}^{\mathrm{p}-1}, \quad \operatorname{det} \mathbf{F}^{*}>0 .
$$

The plastic deformation gradient is, in turn, given by the flow rule

\footnotetext{
${ }^{1}$ Einstein's summation convention implying summation on repeated indices is employed in this paper.
} 


$$
\dot{\mathbf{F}}^{\mathrm{p}}=\mathbf{L}^{\mathrm{p}} \mathbf{F}^{\mathrm{p}}, \quad \mathbf{L}^{\mathrm{p}}=\sum_{\alpha} \dot{\gamma}^{\alpha} \mathbf{S}_{\mathrm{o}}^{\alpha}, \quad \mathbf{S}_{\mathrm{o}}^{\alpha}=\mathbf{m}_{\mathrm{o}}^{\alpha} \otimes \mathbf{n}_{\mathrm{o}}^{\alpha},
$$

where $\mathbf{m}_{\mathrm{o}}^{\alpha}$ and $\mathbf{n}_{\mathrm{o}}^{\alpha}$ are time-independent orthonormal unit vectors which define the slip direction and the slip plane normal of the slip system $\alpha$ in a fixed (initial) reference configuration, respectively, and $\dot{\gamma}^{\alpha}$ is the plastic shearing rate on this slip system. The plastic shearing rate on the slip system $\alpha$ is taken as

$$
\dot{\gamma}^{\alpha}=\dot{\gamma}_{\mathrm{o}}\left|\frac{\tau^{\alpha}}{S^{\alpha}}\right|^{1 / m} \operatorname{sign}\left(\tau^{\alpha}\right), \quad \tau^{\alpha} \cong \mathbf{T}^{*} \cdot \mathbf{S}^{\alpha},
$$

where $\tau^{\alpha}$ and $s^{\alpha}$ are the resolved (Schmid) shear stress and the slip resistance associated with slip system $\alpha, \dot{\gamma}_{\text {o }}$ is a reference value of slip rate (typically set to $0.001 \mathrm{~s}^{-1}$ ), and $m$ is a slip rate-sensitivity parameter (for plastic deformation of metals at low homologous temperatures, this parameter is given a very small value, typically around 0.01 , to approach rate-independent behavior). For the present problem, we are only interested in the yield properties. Consequently, $s^{\alpha}$ for all slip systems was assumed to be same and equal to $s_{\mathrm{o}}$.

The most widely used approach to obtain the response of a polycrystal from the response of the individual grains is to use extended Taylor's assumption of iso-deformation gradient in all of the crystals comprising the polycrystal. Furthermore, if all grains are assumed to be of the same size, the Cauchy stress in the polycrystal can be taken as a simple number average of the Cauchy stresses in the various grains. Therefore, employing these assumptions, the macroscopic Cauchy stress in the polycrystal $(\overline{\mathbf{T}})$ can be expressed as

$$
\overline{\mathbf{T}}=\frac{1}{N_{\mathrm{c}}} \sum_{k=1}^{N} \mathbf{T}^{(k)}
$$

where $N_{\mathrm{c}}$ is the number of crystals in the aggregate, and $\mathbf{T}^{(k)}$ is the Cauchy stress in the crystal [labeled $(k)$ ].

Typically, a specific imposed macroscopic velocity gradient tensor is provided as input to the Taylor-type polycrystal model described above, and the macroscopic stress history is obtained as an output form the model. For the problem at hand, the following velocity gradient tensor can be imposed on a given polycrystal

$$
\overline{\mathbf{L}}=\left[\begin{array}{ccc}
\beta \dot{\varepsilon} & 0 & 0 \\
0 & \alpha \dot{\varepsilon} & 0 \\
0 & 0 & -(\alpha+\beta) \dot{\varepsilon}
\end{array}\right]
$$

where the imposed strain rate $\dot{\varepsilon}$ may be arbitrarily set to $0.001 \mathrm{~s}^{-1}$, and different values are assigned to both $\alpha$ and $\beta$ in the range [0,1]. The stresses obtained from these computations can be interpolated to extract the yield strength of the polycrystal in three specific stress states, namely, uniaxial stress in $x$-direction, uniaxial stress in $y$-direction, and equi-biaxial stress in $x$ - and $y$-directions. Using these three yield strengths in the Hill's anisotropic yield function [Eq. (4)], we can obtain values for three of the anisotropic yield parameters, namely, $G, H$, and $F$. Next, imposing a monotonic simple shear deformation in the $x-y$ plane on a polycrystal and obtaining 
the shear yield of the polycrystal, and using that value in the Hill's anisotropic yield function will provide a value for $N$. Therefore, it is possible to use the Taylor-type polycrystal plasticity model and extract a set of values for all of the required anisotropic yield parameters in Hill's anisotropic yield function as described above. It should be noted that alternative, and more refined, descriptions of yield functions have been proposed by Barlat et al. (1991, 1997), Khan and Cheng (1998), Han et al. (2003) for orthotropic polycrystalline materials. It was, however, found that the results for the present problem were not sensitive to either choice of the yield function.

\section{Microstructure optimization by MSD}

The goal of MSD for the present problem is to identify all possible microstructures within the context of chosen physics and mechanics of the problem that will result in best performance. In the present context, this translates to identifying all possible textures that will lead to best possible performance within the chosen mechanical framework (the upper bound theory for elastic stiffness, the Taylor-type polycrystal code for plastic yield, and the Hill's anisotropic yield function). Note that the number of distinct choices for textures is indeed infinite, and there currently do not exist any alternative methodologies that can potentially find the solution(s) to this inverse problem.

In the MSD framework, we start with a spectral representation of the relevant statistics of the microstructure. For the present problem, this is the orientation distribution function $f(g)$. An appropriate set of orthonormal basis functions, $\ddot{T}_{l}^{m n}(g)$, that reflect the needed crystal and sample symmetries have already been provided by Bunge (1993). Following Bunge, the spectral representation of $f(g)$ can be expressed as

$$
f(g)=\sum_{l=0}^{\infty} \sum_{m=-l}^{+l} \sum_{n=-l}^{+l} F_{l}^{m n} \dot{\bar{T}}_{l}^{m n}(g)
$$

Note that the set of Fourier coefficients $\left\{F_{l}^{m n}\right\}$ in Eq. (15) identify uniquely the function $f(g)$, i.e. there exists a one-to-one correspondence between them.

Procedures to obtain the Fourier coefficients for any given function $f(g)$, using inverse-Fourier transforms, are detailed in Bunge (1993). However, Fourier coefficients corresponding to single crystal orientations are more easily obtained. It should be noted that for a single crystal, the orientation distribution function is correctly represented by a dirac-delta function, and therefore the corresponding Fourier coefficients can be obtained simply by evaluating the basis functions $\dot{\vec{T}}_{l}^{m n}(g)$ at the specific orientation (Bunge, 1993).

The complete Euler space, representing all possible single crystal orientations, was populated with about 1000 evenly distributed single crystal orientations. The Fourier coefficients for this set of 1000 single crystal orientations were computed using the 
methods described above. These coefficients were used to delineate a convex hull that represents all possible polycrystal orientation distribution functions (Adams et al., 2001). This microstructure hull in its first three-dimensional subspace is shown in Fig. 2. The following inherent attributes of the microstructure hull are worth noting: 1. Polycrystals are by definition comprised of single crystals; all polycrystal ODFs are therefore simply a volume weighted average of the constituent single crystal ODFs. This relationship between single crystal states and the universe of possible polycrystal states imparts convexity to spectral representations of the type shown in Fig. 2. The convexity of the microstructure hull in Fig. 2 implies that all possible polycrystal ODFs necessarily reside inside the hull, i.e. polycrystal ODFs corresponding to points outside the hull are impossible.

2. The microstructure hull shown in Fig. 2 is the same for all cubic polycrystals (including all fcc and bec materials).

3. The microstructure hull is shown in Fig. 2 in only its first three-dimensional subspace. Its representation, in reality, extends to infinite dimensions [see Eq. (15)]. Convexity of the microstructure hull described earlier guarantees this convexity in all subspaces. It should, however, be noted that although the representation

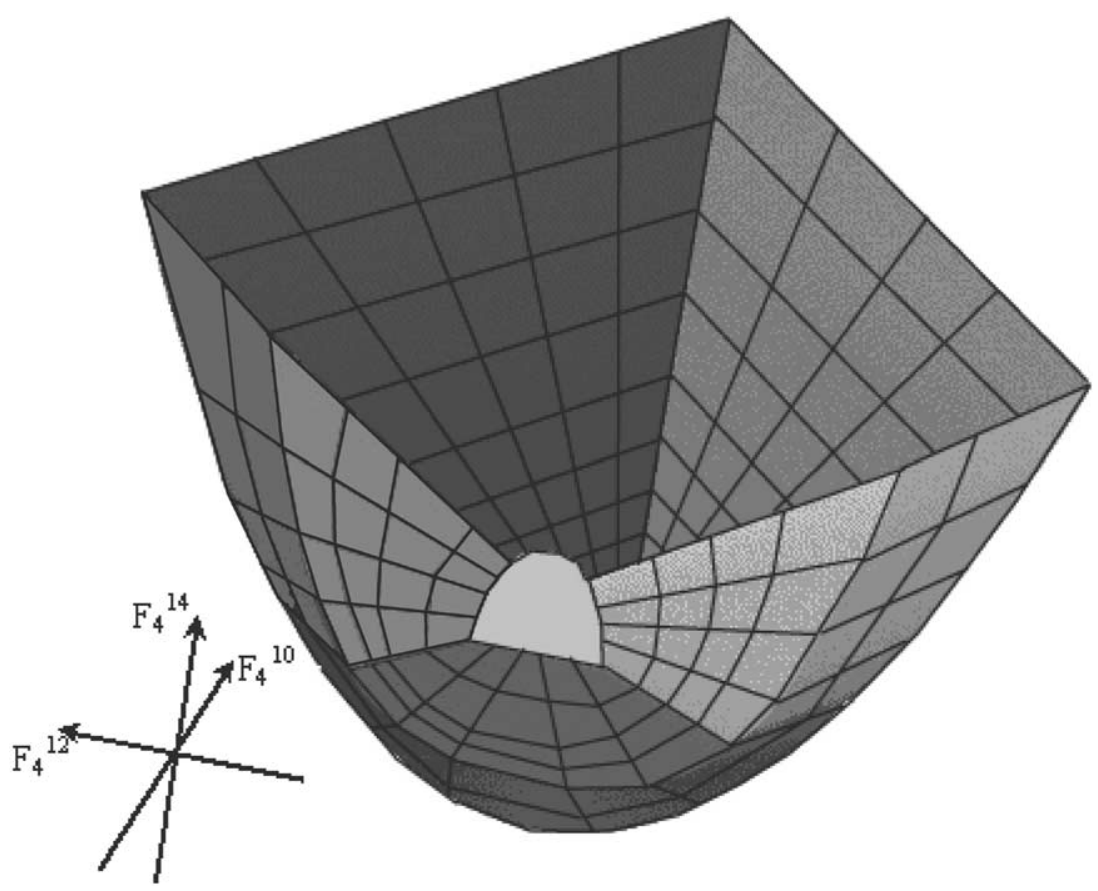

Fig. 2. The microstructure hull for ODFs of cubic polycrystals in the first three dimensions. Only the exterior surface of the hull is shown, with different colors representing different parts of the exterior surface. Furthermore, to improve the clarity of the figure, the top surface encompassing the convex hull has not been shown in this figure. 
of the microstructure hull extends to infinite dimensions, only a finite number of these dimensions control the selected physical or mechanical property of interest to a specific design problem (Adams et al., 2001; Bunge, 1993).

Having identified the microstructure hull, we now turn our attention to representation of properties and performance in this hull. In general, the functional dependence of any tensorial property of a single crystal on its lattice orientation [generically denoted by $P(g)$ ] can be represented in Fourier space as

$$
P(g)=\sum_{l=0}^{\infty} \sum_{m=-l}^{+l} \sum_{n=-l}^{+l} P_{l}^{m n} \dot{\bar{T}}_{l}^{m n}(g)
$$

where $\left\{P_{l}^{m n}\right\}$ represent a set of Fourier coefficients specific to the selected tensorial property. If a simple volume averaging homogenization rule [often corresponding to upper bound theory; see Eq. (8)] is selected for the overall property of the polycrystal, it can be expressed as

$$
\bar{P}=\oiint_{M_{\mathrm{c}}} f(g) P(g) \mathrm{d} g
$$

Substituting Eqs. (15) and (16) in Eq. (17), and exploiting the orthonormality of the basis functions, $\dot{T}_{l}^{m n}(g)$, results in the following desired expression.

$$
\bar{P}=\sum_{l=0}^{\infty} \sum_{m=-l}^{+l} \sum_{n=-l}^{+l} P_{l}^{m n} F_{l}^{m n}
$$

Eq. (18) indicates that if a simple volume averaging homogenization rule is employed, the iso-property surfaces in the Fourier space are strictly hyperplanes Adams et al. (2001).

For the design problem at hand, there are nine independent elastic stiffness parameters (for orthotropic microstructures) that define $\bar{C}_{i j k l}$ [Eq. (8)]. Fourier representations were derived for each of these parameters assuming the single crystal elastic constants of copper $\left(C_{11}=168.4 \mathrm{GPa}, C_{12}=121.4 \mathrm{GPa}, C_{44}=75.4 \mathrm{GPa}\right)$. As an example, the following expression was derived for $\bar{C}_{1111}$ for copper polycrystals.

$$
\bar{C}_{1111}=209.92-20.386 F_{4}^{10}+30.390 F_{4}^{12}-40.202 F_{4}^{14} \quad \mathrm{GPa}
$$

This expression indicates that for the chosen physical framework of the problem, the dependence of elastic properties on the microstructure extends only to the first three dimensions in the Fourier representation. A plot of the maximum percentage error in the Fourier representation as a function of the number of terms in the expression is shown in Fig. 3. For elastic properties, the error goes to zero after the first three terms in the Fourier representation. Note also that the drop in the error is quite steep with the inclusion of additional terms in the Fourier representation, even in these first three terms.

For the yield properties, different velocity gradient tensors of the type described in Eq. (14) were imposed on different single crystal orientations. The resulting functional dependence of each stress component in each crystal on its lattice orientation 


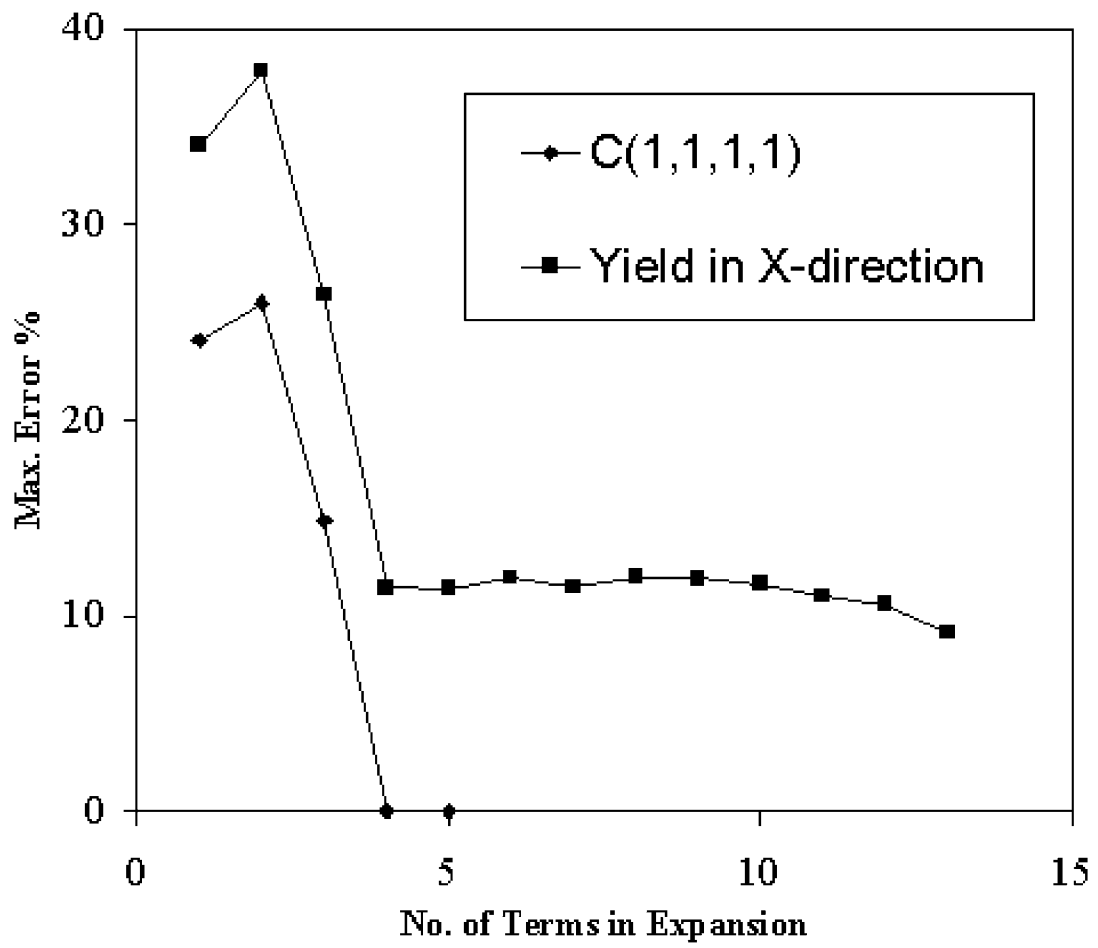

Fig. 3. The variation of the maximum percentage error in the Fourier representation of properties as a function of the number of terms taken into account in the expansion.

was expressed in Fourier space by linear regression techniques for each choice of the velocity gradient tensor. By virtue of the Taylor assumption described above, the overall stress state in the polycrystal is given by

$$
\bar{\sigma}_{i j}=\oiint_{M_{c}} f(g) \sigma_{i j}(g) \mathrm{d} g
$$

Using this approach, we have developed expressions for the overall stress states in the polycrystal as a function of the microstructure for different choices of $\alpha$ and $\beta$ in Eq. (14), and numerically interpolated these results to find the yield strength of the polycrystal in the three specific stress states needed for the present design problem (uniaxial tension in $x$-direction, uniaxial tension in $y$-direction, equi-biaxial tension in $x$-and $y$-directions). Fig. 3 shows a plot of the maximum error in prediction of the yield strength in $x$-direction using Fourier representations as a function of the number of terms used in the expansion. Although a sharp decrease in error is observed with the inclusion of the first three terms, thereafter the decrease in error with additional terms is rather slow. For the chosen rate-sensitivity parameter $[m=0.01$ in Eq. (12)] in this example, it can be shown theoretically that the error in this 
representation will go to zero by considering all terms up to $l=202$ in the Fourier expansion [Eq. (15)]. However, note that the maximum error is plotted in Fig. 3, and a maximum error of $10 \%$ is quite reasonable for the present case study. Therefore, the rest of the analysis is carried out with only the first five terms in the expansion.

Having established the functional dependence of the required elastic and plastic parameters with the microstructure in the Fourier space, it is relatively easy to search through the microstructure hull to find the locations of optimal overall performance. For the present case, the search was done by first binning (in five dimensions) the microstructure hull coarsely, and then refining the discretization in the areas of interest to further narrow down the location of best performance. The solutions in Fourier space corresponding to best and worst performance are shown in Table 1.

The solutions described in Table 1 indicate that the best performance corresponds to an improvement of about $59 \%$ in load carrying capacity compared to the worst case. This can have a major effect on service life of components in fatigue loading conditions. Another significant result from the present analysis that the random microstructure (corresponds to origin in the microstructure hull) has a performance of about $1.02 \sigma_{\mathrm{o}}$, which is significantly closer to the best performance compared to the worst performance. A far-reaching implication of this result is that

Table 1

Summary of MSD predictions for best and worst load carrying capacities

\begin{tabular}{lllllll}
\hline & Load carrying capacity & $F_{4}^{10}$ & $F_{4}^{12}$ & $F_{4}^{14}$ & $F_{6}^{10}$ & $F_{6}^{12}$ \\
\hline Best performance & $\sigma^{\infty}=1.065 s_{\mathrm{o}}$ & -0.35 & -0.11 & -0.15 & -0.35 & +0.34 \\
Worst performance & $\sigma^{\infty}=0.67 s_{\mathrm{o}}$ & +0.55 & -0.15 & -0.55 & -0.15 & +0.05 \\
\hline
\end{tabular}

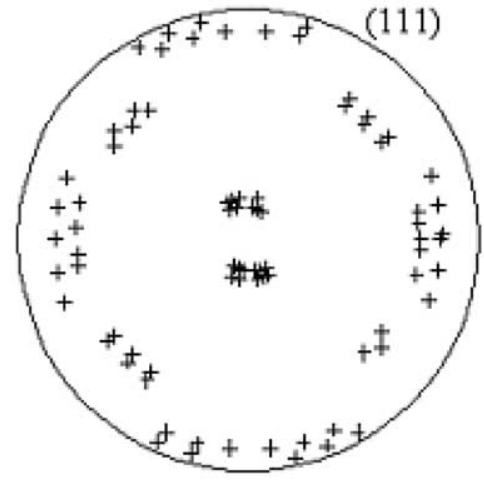

(a) Best Performance

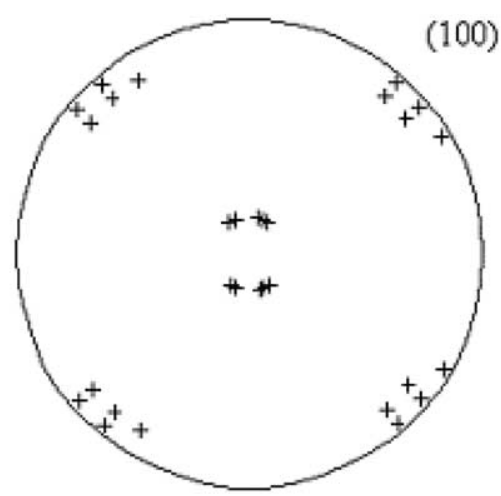

(b) Worst Performance

Fig. 4. Pole figures of the textures identified that lie close to the performances predicted by MSD (see Table 1). 
a much larger number of microstructures are likely to perform worse than the random microstructure compared to those that perform better than the random microstructure. It is especially important that designers understand this result, because in many designs the properties corresponding to random microstructure (isotropic assumption) are often utilized, however the real microstructures used are seldom random.

By repeated trials, we have identified two specific textures that lie somewhat close to the two locations identified in Table 1, respectively. The (111) pole figure of a texture whose Fourier representation lies at $(-0.38,-0.13,-0.13,-0.36,0.30)$ is shown in Fig. 4(a). Note that this location is close to the location that has been predicted to exhibit the best possible performance in Table 1. Note that this texture can be identified as being close to a (111) fiber texture normal to the plane of the plate. Fig. 4(b) shows the (100) pole figure of another texture whose Fourier representation lies at $(0.575,-0.06,-0.55,-0.18,0.16)$. Note that this texture is somewhat close to the location corresponding to worst performance in the Fourier space, and this texture can be identified as being close to a cube texture rotated by $45^{\circ}$ in the plane of the plate.

\section{Verification of MSD predictions}

One of the major objectives of the present study was to verify the MSD predictions with direct comparison against corresponding finite element predictions. A

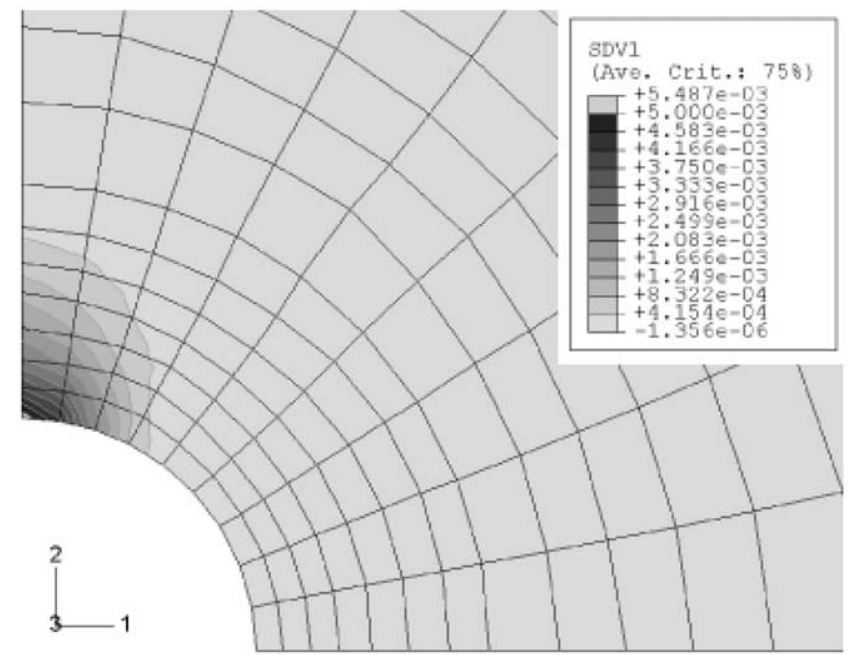

Fig. 5. The finite element mesh of the quarter of the orthotropic plate with a circular hole in the center. The mesh consists of 384 three-dimensional solid elements, and an elastic-viscoplastic Taylor-type crystal constitutive model was employed at each integration point in the mesh. The contours shown correspond to the accumulated local plastic strain at yield. 
three-dimensional finite model consisting of 384 eight-noded linear solid elements was constructed (see the mesh in Fig. 5). Only one-quarter of the square thin plate was simulated and appropriate symmetric boundary conditions were imposed. A uniform displacement boundary condition was imposed on the edge of the plate and plastic strain contours around the hole were monitored carefully to detect initiation of localized yielding in the sample. The geometry for the numerical model was selected such that the hole radius was $1 / 100$ of the plate width and the thickness of the plate was $1 / 10$ of the hole radius. The mesh employed a much higher spatial resolution near the hole and two layers of elements were used in the thickness direction.

The simulation was performed in ABAQUS using a UMAT (user defined material subroutine) that incorporates the Taylor-type elastic-viscoplastic polycrystal model described earlier at each integration point in the mesh. Details of the numerical procedures used and the validation of these procedures can be found in several previous publications of one of the authors (Bronkhorst et al., 1992; Kalidindi and Anand, 1994; Kalidindi et al., 1992; Van Houtte et al., 2002). Simulations were carried out for the specific textures shown in Fig. 4. Each integration point in the finite element mesh was assigned the selected polycrystalline, and the Taylor-type model described earlier was implemented to represent the material constitutive response at each integration point. Typical contours representing the accumulated plastic strain at the initiation of localized yielding in the vicinity of the hole are shown in Fig. 5.

In the finite element simulations, the evolution of the equivalent plastic strain around the hole was monitored, and the load carrying capacity was assumed to have been reached when the maximum local equivalent plastic strain reached a value of 0.005 . The contours of the accumulated plastic strain were similar for both textures. Interestingly, we find the stress concentration factors for both textures to be significantly lower than the stress concentration factor for a random texture. Further, it was also noted that the stress concentration factor was actually slightly higher for the texture that produced the best performance compared to the texture that produced the worst overall performance. This means that the slight increase in the stress concentration factor was more than offset by the much larger increase in the yield strength associated with the solution corresponding to the best performance. The ratio of the predicted load carrying capacities for the two different textures from the finite element simulations was 1.45 . The ratio of the predicted load carrying capacities from the MSD analyses for these specific textures was 1.54. These numbers indicate that the MSD predictions are reasonably accurate. Note that we have accepted some errors in the estimation of the plastic yield properties by limiting the number of terms in the Fourier expansion to the first five terms. The accuracy of the MSD analyses can be improved, as needed, through consideration of the higher order terms in the Fourier expansion. It should also be noted that there are some approximations in the analytical solution provided by Lekhnitskii (1968), and these can also contribute to the differences observed here between the MSD predictions and the finite element predictions. 


\section{Conclusions}

MSD provides an efficient framework for solving inverse problems in materials design. For a selected mechanical framework and a choice of microstructural design variables, MSD provides a framework to identify the complete set of microstructures that are predicted to maximize the performance criterion, while satisfying the design constraints. In the present paper, this was demonstrated in the design of an orthotropic thin plate subjected to an in-plane tensile load and made of polycrystalline copper material. The chosen mechanical framework comprised of the upper bound theory for elastic stiffness, the Taylor-type polycrystal code for plastic yield, and the Hill's anisotropic yield function was selected. The chosen microstructural design variable was the orientation distribution function (ODF). For this problem, it was found that the best performance was about $59 \%$ better than the worst performance, and corresponded to a (111) fiber texture parallel to the plate thickness. These results were confirmed by finite element simulations that employed the exact same mechanical framework and the same description of the microstructure.

\section{Acknowledgements}

Financial support for this work was provided by the Army Research Office, Proposal No. 42566-MS, Dr. David Stepp, Program Director and Army Research Lab, Dr. Scott Schoenfeld, Point of Contact.

\section{References}

Adams, B.L., Henrie, A., Henrie, B., et al., 2001. Microstructure-sensitive design of a compliant beam. Journal of Mechanics and Physics of Solids 49, 1639-1663.

Barlat, F., Becker, R.C., Brem, J.C., et al., 1997. Yielding description for solution strengthened aluminum alloys. International Journal of Plasticity 13, 385-401.

Barlat, F., Lege, D.J., Brem, J.C., 1991. A six-component yield function for anisotropic materials. International Journal of Plasticity 7, 693-712.

Bronkhorst, C.A., Kalidindi, S.R., Anand, L., 1992. Polycrystalline Plasticity and the Evolution of Crystallographic Texture in Face-Centered Cubic Metals. Philosophical Transactions of the Royal Society of London A 341, 443-477.

Bunge, H.-J., 1993. In: Texture Analysis in Materials Science. Mathematical Methods. Cuvillier Verlag, Göttingen.

Alexander, W. (Ed.), 2001. In: CRC Materials Science and Engineering Handbook. CRC Press, Boca Raton.

Han, C.-S., Chung, K., Wagoner, R.H., et al., 2003. A multiplicative finite elasto-plastic formulation with anisotropic yield functions. International Journal of Plasticity 19, 197-211.

Harper, C. (Ed.), Handbook of Materials for Product Design. McGraw-Hill Professional, 2001.

Kalidindi, S.R., Anand, L., 1994. Macroscopic shape change and evolution of crystallographic texture in pre-textured FCC metals. Journal of Mechanics and Physics of Solids 42, 459-490.

Kalidindi, S.R., Bronkhorst, C.A., Anand, L., 1992. Crystallographic texture evolution in bulk deformation processing of FCC metals. Journal of Mechanics and Physics of Solids 40, 537-569. 
Khan, A.S., Cheng, P., 1998. An anisotropic elastic-plastic constitutive model for single and polycrystalline metal. II-experiments and predictions concerning thin-walled tubular OFHC copper. International Journal of Plasticity 14, 209-226.

Lekhnitskii, S. G., 1968. In: Anisotropic Plates. Gordon and Breach, p. 534.

Jones, R.M. (Ed.), 1999. Mechanics of Composite Materials.

Olsen, G.B., 1997. Computational design of hierachically structured materials. Science 277, 1237-1242.

Olsen, G.B., 2000. Pathways of discovery designing a new material world. Science 228, 933-998.

Committee, SoAEFDaE (Eds.), 1997. SAE Fatigue Design Handbook.

Van Houtte, P., Delannay, L., Kalidindi, S.R., 2002. Comparison of two grain interaction models for polycrystal plasticity and deformation texture prediction. International Journal of Plasticity 18, 359377. 